DOI: 10.12731/wsd-2018-4-223-244

УДК 616.12-008.331.1: 616-08-039.32

\title{
ЧАСТОТА ВСТРЕЧАЕМОСТИ, СТРУКТУРА АРТЕРИАЛЬНОЙ ГИПЕРТОНИИ И КОМПЛАЕНТНОСТЬ СРЕДИ ВРАЧЕЙ КРУПНОЙ КЛИНИЧЕСКОЙ БОЛЬНИЦЫ ГОРОДА КРАСНОЯРСКА
}

\author{
Деревянных Е.В., Балашова Н.А., Яскевич Р.А., \\ Москаленко О.Л.
}

Цель. Изучение частоты встречаемости, структуры артериальной гипертонии (АГ) и комплаентности среди врачей крупной клинической больнищьы г. Красноярска.

Материалы и методы. Обследовано 317 врачей в возрасте от 23 до 77 лет. Обследование включало анкетирование, клинические, инструментальные, функииональные и лабораторные методы исследования, изучение приверженности к лечению АГ.

Результаты. По данным проведенного исследования частота встречаемости АГ среди обследованных врачей составила 43,2\% и не превышала среднероссийский уровень. Данный показатель был существенно выше среди мужчин врачей, в сравнении с женщинами. Указанное гендерное отличие особенно выражено в возрастной группе 20-29 лет и 30-39 лет. Среди анестезиологов, реаниматологов и врачей лучевой диагностики АГ встречалась чаще по сравнению с врачами других специальностей. У врачей не зависимо от пола чаще отмечалась АГ 1 степени. Несмотря на достаточно высокую информированность о методах профилактики и лечения сердечно-сосудистых заболеваний, показатели приверженности к лечению АГ были ниже среднероссийского уровня. Врачи хирургических отделений имели самые низкие показатели осведомленности и комплаентности, в сравнении с врачами других специальностей.

Заключение. Результаты проведенного исследования позволяют сделать выводы о высокой распространенности АГ среди врачей.

Ключевые слова: врачи; артериальная гипертония; комплаентность; осведомленность. 


\title{
FREQUENCY OF MEETING, STRUCTURE OF ARTERIAL HYPERTENSION AND COMPLICATION AMONG DOCTORS OF LARGE CLINICAL HOSPITAL OF KRASNOYARSK CITY
}

\author{
Derevyannich E.V., Balashova N.A., Yaskevich R.A., \\ Moskalenko O.L.
}

The purpose of the study. Study of incidence, structure of arterial hypertension and compliance among doctors of a large clinical hospital in Krasnoyarsk.

Materials and methods. A total of 317 physicians aged 23 to 77 years were examined. The examination included a questionnaire, clinical, instrumental, functional and laboratory methods of research, the study of adherence to the treatment of hypertension.

Results. According to the study, the incidence of hypertension among the examined doctors was $43.2 \%$ and did not exceed the average Russian level. This figure was significantly higher among male doctors compared to women. This gender difference is particularly pronounced in the age group 20-29 years and 30-39 years. Among anesthesiologists, intensive care specialists and physicians radiological diagnosis of hypertension was more common compared with physicians of other specialties. The doctors regardless of sex were more often noted grade 1 hypertension. Despite the rather high awareness of methods of prevention and treatment of cardiovascular diseases, received in the framework of professional education, the indicators of adherence to the treatment of hypertension were lower than the average Russian level. Doctors of surgical departments had the lowest indicators of awareness and compliance, in comparison with doctors of other specialties.

Conclusion. The results of the study allow to draw conclusions about the high prevalence of hypertension among doctors.

Keywords: doctors; arterial hypertension; compliance; awareness.

\section{Введение}

Несмотря на некоторый прогресс в профилактике и лечении хронических неинфекционных заболеваний (ХНИЗ), болезни системы кровообращения (БСК) остаются ведущей причиной заболеваемости, инвалидности и смертности во многих странах мира [29, эл. ресурс; 31 , с. 483-7], в том числе и в России [2, с. 4-14; 3, с. 551-559; 19, с. 123-125; 28, с. 17:138]. 
Артериальная гипертония (АГ) является одним из важнейших факторов риска сердечно-сосудистых заболеваний (СС3) [2, с. 4-143; 19, с. 123-125; 28, с. 17:138]. По оценкам экспертов Всемирной организации здравоохранения (ВО3), около 25\% взрослого населения в мире имеет АГ, и этот показатель, по прогнозам, увеличится к 2025 году до 29\% [32, эл. ресурс]. В формировании стратегии профилактики АГ, изучение региональных особенностей распространенности и выраженности факторов риска (ФР) АГ [3, с. $551-559 ; 18$, с. 92-95; 19, с. $123-125,34$, с. 15], является одним из ключевых вопросов при разработке эффективных систем скрининга и контроля АГ среди населения различных регионов страны и в том числе среди жителей Сибири и Крайнего Севера [17, с. 55-57; 18, с. 92-95; 24, c. 94-96; 26, с. 41-58; 27, с. 346-348; 33, с. 59-73].

Результаты проведенных исследований свидетельствует о недостаточной первичной профилактике АГ, как на популяционном уровне, так и среди лиц высокого риска [4, с. 285-289; 5, с. 290-295; 23, эл. ресурс]. Среди причин такой ситуации - недостаточная приверженность пациентов АГ к терапии [4, с. 285-289; 10, с. 48-52; 11, с. 21-23; 20, эл. ресурс]. Одним из важнейших факторов формирования здоровья населения, является состояние здравоохранения и здоровья его работников, призванных укреплять и поддерживать здоровье всего населения страны [12, с. 137 $144 ; 13$, с. $54-58 ; 14$, с. $12-24 ; 15$, с. 38-42; 22, с. 5-9]. Именно поэтому состоянию здоровья медицинских работников в последнее время уделяется повышенное внимание [9, с. 26-46; 16, с. 72-82; 21, с. $126-131 ; 25$, с. 28 $32 ; 30$, с. 1659-1662; 31, с. 483-7], при этом существующие исследования в основном посвящены изучению распространенности среди медицинского персонала сердечно-сосудистой патологии [1, с. 52-5; 6, с. 10-33; 7, с. 89-92; 8, с. 130-139]. В связи с этим большой интерес представляют данные о частоте встречаемости АГ, особенностях приверженности к лечению среди врачей крупной городской клинической больницы.

\section{Цель работы}

Изучить частоту встречаемости, структуру артериальной гипертонии и комплаентность среди врачей крупной клинической больницы города Красноярска.

\section{Объект и методы исследования}

Объектом исследования были врачи крупной клинической больницы города Красноярска. Всего обследовано 317 пациентов, средний возраст 
41,9 [95\%ДИ: 40,5-43,4] года, из них 142 женщины (средний возраст: 41,2 [95\%ДИ: 39,4-42,9] года) и 175 мужчин (средний возраст: 42,9 [95\%ДИ: $40,5-45,3]$ года) $(\mathrm{p}=0,57)$.

Протокол исследования включал: осмотр, интервьюирование, анкетирование, двукратное измерение артериального давления, эхокардиографию, электрокардиографию, антропометрию, определение биохимических показателей сыворотки крови.

Приверженность к лечению АГ оценивали по общепринятой методике Мориски-Грина (Morisky D. et al., 1986) которая включает 4 вопроса. Каждый ответ «нет» оценивался в 1 балл. Комплаентными (приверженными) считались больные, набравшие 4 балла. Больные, набравшие 2 балла, и менее считались неприверженными. Больные, набравшие 3 балла, считаются недостаточно приверженными и находящимися в группе риска по развитию неприверженности.

Статистическая обработка результатов исследования осуществлялась с помощью пакета программ Statistica 6.1. Полученные данные представлены в виде средней величины и доверительного интервала. Две независимые группы сравнивались с помощью U-критерия Манна-Уитни, анализ различия частот в двух независимых группах проводился при помощи точного критерия Фишера с двусторонней доверительной вероятностью, критерия $\chi^{2}$ с поправкой Йетса. Статистически значимыми считали различия при $\mathrm{p}<0,05$.

\section{Результаты исследования}

Среди обследованных врачей частота встречаемости АГ составила 43,22\% [95\%ДИ: 37,76-49,00], что не превышает среднероссийский уровень $(44,0 \%)$ [2, с. 4-14] и ниже показателей распространенность АГ в Красноярском крае - 49,4\% [3, с. 551-559]. Среди обследованных мужчин частота встречаемости АГ была выше: 48,6\% [95\%ДИ: 41,17-48,57] в сравнении с женщинами 36,6\% [95\%ДИ: 28,70-36,62] ( $=0,021)$. Полученные результаты о распространенности АГ среди врачей сопоставимы с данными Карамновой Н.С. с соавт. (2009), согласно которых распространенность АГ среди обследованных составила 44\% [13, с. 54-58] и с результатами, полученными в ходе проведенных исследования среди врачей, работающих в ЛПУ г. Ростова на Дону и области, $44 \%$ среди мужчин и $31,2 \%$ у женщин [22, с. 5-9]. Как показали результаты исследования Шальновой С.А. и соавт. (2008), российские врачи-мужчины страдают АГ в $32,1 \%$, женщины - 30,3\% [25, с. 28-32]. 
При сравнении исследуемых групп в зависимости от возраста установлено, что мужчины в возрасте 20-29 лет $(25,0 \%$ 95\%ДИ: 10,86-25,00) и 30-39 лет $(38,9 \% 95 \%$ ДИ: $25,89-46,74)$ болеют чаще АГ, чем женщины $(9,1 \%$ 95\%ДИ: $0,49-14,17)(\mathrm{p}=0,05)$ и $(2,8 \% 95 \%$ ДИ: $3,14-23,57)(\mathrm{p}=0,003)$ (табл. 1), т.е. становление АГ начинается раньше. Наибольшая частота встречаемости АГ среди женщин отмечена в возрасте $50-59$ лет $(80,0 \%$ 95\%ДИ: 64,32-80,0) и 60 лет и старше $(69,7 \%$ 95\%ДИ: 50,76-69,57) соответственно.

Таблицуа 1.

Частота встречаемости АГ среди мужчин и женщин

в различных возрастных группах

\begin{tabular}{|c|c|c|c|c|c|c|c|}
\hline \multirow{2}{*}{$\begin{array}{c}\text { Возраст- } \\
\text { ные груп- } \\
\text { пы (лет) }\end{array}$} & \multicolumn{2}{|c|}{ Всего } & \multicolumn{2}{|c|}{ Мужчины } & \multicolumn{2}{|c|}{ Женщины } & \multirow[b]{2}{*}{$p$} \\
\hline & Абс. & $\%$ & Абс. & $\%$ & Абс. & $\%$ & \\
\hline 1. $20-29$ & 10 & 7,3 & 9 & 25,0 & 1 & 2,8 & $\begin{array}{c}p_{\mathrm{M}, \mathrm{*}}= \\
0,05\end{array}$ \\
\hline 2. $30-39$ & 24 & 17,5 & 21 & 38,9 & 3 & 9,1 & $\begin{array}{l}p_{\mathrm{M}, \mathrm{w}}= \\
0,003\end{array}$ \\
\hline 3. $40-49$ & 40 & 29,2 & 28 & 63,6 & 12 & 48,0 & $\begin{array}{c}p_{\mathrm{M}, * \pi}= \\
0,15\end{array}$ \\
\hline 4. $50-59$ & 35 & 25,5 & 15 & 55,6 & 20 & 80,0 & $\begin{array}{l}p_{\mathrm{M}, \%}= \\
0,006\end{array}$ \\
\hline $\begin{array}{l}5.60 \\
\text { и старше }\end{array}$ & 28 & 20,4 & 12 & 85,7 & 16 & 69,7 & $\begin{array}{c}p_{\mathrm{M}, \%}= \\
0,02\end{array}$ \\
\hline$p$ & $\begin{array}{l}\mathrm{p}_{1,2}=0,008 \\
\mathrm{p}_{1,3}=0,0001 \\
\mathrm{p}_{1,4}=0,0001 \\
\mathrm{p}_{1,5}=0,001 \\
\mathrm{p}_{2,3}=0,016\end{array}$ & $\begin{array}{l}\mathrm{p}_{2,4}=0,071 \\
\mathrm{p}_{2,5}=0,322 \\
\mathrm{p}_{3,4}=0,294 \\
\mathrm{p}_{3,5}=0,062 \\
\mathrm{p}_{4,5}=0,195\end{array}$ & $\begin{array}{l}\mathrm{p}_{1,2}=0,013 \\
\mathrm{p}_{1,3}=0,0003 \\
\mathrm{p}_{1,4}=0,135 \\
\mathrm{p}_{1,5}=0,321 \\
\mathrm{p}_{2,3}=0,155\end{array}$ & $\begin{array}{l}\mathrm{p}_{2,4}=0,174 \\
\mathrm{p}_{2,5}=0,06 \\
\mathrm{p}_{3,4}=0,017 \\
\mathrm{p}_{3,5}=0,003 \\
\mathrm{p}_{4,5}=0,338\end{array}$ & $\begin{array}{l}\mathrm{p}_{1,2}=0,309 \\
\mathrm{p}_{1,3}=0,001 \\
\mathrm{p}_{1,4}=0,0001 \\
\mathrm{p}_{1,5}=0,0001 \\
\mathrm{p}_{2,3}=0,011\end{array}$ & $\begin{array}{l}\mathrm{p}_{2,4}=0,0001 \\
\mathrm{p}_{2,5}=0,001 \\
\mathrm{p}_{3,4}=0,068 \\
\mathrm{p}_{3,5}=0,254 \\
\mathrm{p}_{4,5}=0,268\end{array}$ & \\
\hline
\end{tabular}

Резюмируя вышеизложенное следует отметить, что о у мужчин врачей АГ регистрируется значительно чаще (48,6\% против $36,6 \%)$, особенно в более молодом возрасте (20-29 лет и 30-39 лет) в сравнении с врачами женщинами, что согласуются с общероссийской тенденцией [2, с. 4-14; 3, c. 551-559]. Так по данным эпидемиологического исследования ЭССЕ-РФ, распространенность артериальной гипертензии в Красноярском крае у мужчин была выше $(56,3 \%$ против $43,7 \%)$ и закономерно увеличивалась с возрастом (с 30,4\% до 72,1\%) [3, с. 551-559]. Указанное гендерное отличие особенно было выражено в самой молодой возрастной группе 25-34 года, однако по мере приближения к возрасту 65 лет распространенность 
АГ среди мужчин и женщин нивелировалась за счет опережающего прироста числа больных АГ среди женщин [3, с. 551-559]. Распределение величины АД у участников исследования в Томской области также показало четкую зависимость АД от возраста обследуемых [15, с. 38-42]. Выявлена положительная корреляционная зависимость между возрастом медработников и уровнем систолического $(\mathrm{p}=0,436)$ и диастолического АД ( $\mathrm{p}=0,297)$. Наибольшее число лиц с АГ и повышенным АД (высокое нормальное АД) выявлено в группе лиц старше 50 лет [15, с. 38-42].

При анализе частоты встречаемости АГ среди врачей различных специальностей установлено, что среди анестезиологов, реаниматологов и врачей лучевой диагностики АГ встречалась чаще по сравнению с врачами других специальностей (табл. 2).

Таблицуа 2 .

Частота встречаемости АГ у врачей различных специальностей

\begin{tabular}{|c|c|c|c|c|c|c|c|}
\hline \multirow{2}{*}{ Врачи } & \multicolumn{2}{|c|}{ Всего } & \multicolumn{2}{|c|}{ Мужчины } & \multicolumn{2}{|c|}{ Женщины } & \multirow[b]{2}{*}{$p$} \\
\hline & Абс. & $\%$ & Абс. & $\%$ & Абс. & $\%$ & \\
\hline 1. Терапевты & 43 & 34,4 & 10 & 25 & 33 & 38,8 & $\begin{array}{l}p_{\mathrm{M}, \%}= \\
0,117\end{array}$ \\
\hline 2. Хирурги & 65 & 46,1 & 54 & 52,9 & 11 & 28,2 & $\begin{array}{l}p_{\mathrm{M}, \mathrm{K}}= \\
0,007\end{array}$ \\
\hline $\begin{array}{l}\text { 3. Анестези- } \\
\text { олог. и реа- } \\
\text { ниматолог. }\end{array}$ & 20 & 57,1 & 13 & 59,1 & 7 & 53,8 & $\begin{array}{l}p_{\mathrm{M}, \mathrm{K}}= \\
0,518\end{array}$ \\
\hline $\begin{array}{l}\text { 4. Лучевой } \\
\text { диагностики }\end{array}$ & 9 & 56,2 & 8 & 73 & 1 & 20 & $\begin{array}{l}p_{\mathrm{M}, \mathrm{K}}= \\
0,043\end{array}$ \\
\hline$p$ & $\begin{array}{l}\mathrm{p}_{1,2}=0,005 \\
\mathrm{p}_{1,3}=0,001 \\
\mathrm{p}_{1,4}=0,0001\end{array}$ & $\begin{array}{l}\mathrm{p}_{2,3}=0,0001 \\
\mathrm{p}_{2,4}=0,0001 \\
\mathrm{p}_{3,4}=0,024\end{array}$ & $\begin{array}{l}\mathrm{p}_{1,2}=0,0001 \\
\mathrm{p}_{1,3}=0,327 \\
\mathrm{p}_{1,4}=0,402\end{array}$ & \begin{tabular}{|l|}
$\mathrm{p}_{2,3}=0,0001$ \\
$\mathrm{p}_{2,4}=0,0001$ \\
$\mathrm{p}_{3,4}=0,176$
\end{tabular} & $\begin{array}{l}\mathrm{p}_{1,2}=0,0001 \\
\mathrm{p}_{1,3}=0,0001 \\
\mathrm{p}_{1,4}=0,0001\end{array}$ & $\begin{array}{l}\mathrm{p}_{2,3}=0,218 \\
\mathrm{p}_{2,4}=0,002 \\
\mathrm{p}_{3,4}=0,029\end{array}$ & \\
\hline
\end{tabular}

Проводился анализ частоты встречаемости АГ среди врачей различных специальностей в зависимости от пола (табл. 2). Среди врачей терапевтов мужчин и женщин значимых отличий в частоте встречаемости АГ не выявлено, однако наблюдалась тенденция в большей частоте встречаемости АГ у женщин 38,8\% [95\%ДИ: 28,46-38,82] против 25\% [95\%ДИ: $11,58-25,00 \%]$ у мужчин $(\mathrm{p}=0,15)$. У анестезиологов АГ наблюдалась одинаково часто как у мужчин, так и у женщин и составила 59,1\% [95\%ДИ: $38,55-59,09]$ и 53,8\% [95\%ДИ: 26,75-53,85] соответственно (p=0,9). Врачи хирурги мужчины чаще страдают АГ, чем женщины: 51,9\% [95\%ДИ: 43,25-52,94] против 28,2\% [95\%ДИ: 14,08-28,2] ( $=0,01)$ соответствен- 
но. Аналогичные данные были получены при сравнении врачей лучевой диагностики: у мужчин 72,7\% [95\%ДИ: 43,44-90,25] и женщин $20 \%$ [95\%ДИ: 3,62-62,45] соответственно ( $\mathrm{p}=0,043)$. По полученным данным частота встречаемости АГ была выше у врачей мужчин хирургов и лучевой диагностики в сравнении с врачами женщинами.

При сравнении средних значений систолического и диастолического АД между мужчинами и женщинами установлено, что у мужчин уровни САД и ДАД было выше и составили для САД: 145,53 [95\%ДИ: 142,71-148,35] мм рт. ст. против 140,42 [95\%ДИ: 134,47-146,38] мм рт. ст. ( $\mathrm{p}=0,054)$, для ДАД: 92,89 [95\%ДИ: 90,90-94,88] мм рт.ст. и 88,11 [95\%ДИ: 85,01-91,22] мм рт. ст. ( $\mathrm{p}=0,006)$ соответственно.

Среди врачей терапевтов у мужчин среднее САД составляет 146,61 [95\%ДИ: 137,24-155,99] рт.ст., ДАД 95,38 [95\%ДИ: 88,79-101,97] мм рт.ст., у женщин 142,29 [95\%ДИ: 122,76-161,81] мм рт.ст., ДАД 88,57 [95\%ДИ: 78,97-98,17] мм.рт.ст. $(\mathrm{p}=0,19)$. Среди врачей хирургов у мужчин САД 144,40 [95\%ДИ: 135,33-153,47] мм рт.ст., ДАД 89,40 [95\%ДИ: 83,22-95,58] мм рт.ст., у женщин САД 139,64 [95\%ДИ: 131,31-147,96] мм рт.ст., ДАД 88,42 [95\%ДИ: 83,94-92,91] мм рт.ст. ( $=0,001)$. Среди мужчин врачей анестезиологов и реаниматологов САД 145,07 [95\%ДИ: 141,89-148,25] мм рт.ст., ДАД 92,96 [95\%ДИ: 90,46-95,46] мм рт.ст., у женщин 140,91 [95\%ДИ: 129,42-152,39] мм рт.ст., ДАД 86,54 [95\%ДИ: 81,62-91,47] мм рт.ст. $(\mathrm{p}=0,12)$. Среди врачей лучевой диагностики у мужчин САД 148,25 [95\%ДИ: 131,66-164,84] мм рт.ст., ДАД 92,75 [95\%ДИ: 86,37-99,13] мм рт.ст., у женщин 148 мм рт.ст., ДАД 92,0 мм рт.ст. (p=0,12).

У врачей мужчин хирургов значения САД и ДАД выше, чем у врачей женщин хирургов. Гендерных различий среди врачей других специальностей по уровням АД не выявлено.

При распределении АД в зависимости от уровня в соответствии с классификацией (ВОЗ/МОАГ 2013 г.) у врачей не зависимо от пола чаще отмечалась АГ 1 степени (48,2\% 95\%ДИ: 39,81-48,18) (p=0,001) (табл. 3).

Осведомлённость о наличии АГ среди обследованных врачей составила 67,15\% [95\%ДИ: 57,56-67,15]. Наибольшая осведомленность среди обследованных приходилась на возрастную группу 50-59 лет (80,0\% 95\%ДИ: $66,7-80,0)$ и 60 лет и старше $(89,3 \%$ 95\%ДИ: 77,8-89,3) (табл. 4).

Среди женщин показатель осведомленности о заболевании был выше, чем у мужчин: 86,54\% [95\%ДИ: 76,57-86,54] и 55,29\% [95\%ДИ: 41,08$73,82](\mathrm{p}=0,001)$ соответственно. У женщин наибольшая осведомленность приходилась на возрастные группы 50-59 лет (85,0\% 95\%ДИ: 69,3-85,0) 
и 60 лет и старше $(100,0 \%$ 95\%ДИ: 80,6-100,0) и была выше в сравнении с мужчинами в этих возрастных группах: (73,3\% 95\%ДИ: 50,9-73,3) $(\mathrm{p}=0,006)$ и $(75,0 \%$ 95\%ДИ: $50,5-75,0)(\mathrm{p}=0,044)$ соответственно (табл. 4).

Таблица 3.

Распределение АД в зависимости от уровня в соответствии с классификацией АГ

\begin{tabular}{|c|c|c|c|c|c|c|c|}
\hline & \multicolumn{2}{|c|}{ Всего } & \multicolumn{2}{|c|}{ Мужчины } & \multicolumn{2}{|c|}{ Женщины } & \multirow[b]{2}{*}{$p$} \\
\hline & Абс. & $\%$ & Абс. & $\%$ & Абс. & $\%$ & \\
\hline 1. АГ 1 ст. & 66 & 48,2 & 39 & 45,9 & 27 & 51,9 & $\begin{array}{l}p_{\mathrm{M}, \mathrm{K}}= \\
0,305\end{array}$ \\
\hline 2. АГ 2 ст. & 39 & 28,5 & 25 & 29,4 & 14 & 26,9 & $\begin{array}{l}p_{\mathrm{M}, \pi}= \\
0,456\end{array}$ \\
\hline 3. АГ 3 ст. & 17 & 12,4 & 12 & 14,1 & 5 & 9,6 & $\begin{array}{l}p_{\mathrm{M}, \mathrm{*}}= \\
0,310\end{array}$ \\
\hline 4. ИСАГ & 15 & 10,9 & 9 & 10,6 & 6 & 11,5 & $\begin{array}{l}p_{\mathrm{M}, \pi}= \\
0,536\end{array}$ \\
\hline$p$ & $\begin{array}{l}\mathrm{p}_{1,2}=0,001 \\
\mathrm{p}_{1,3}=0,0001 \\
\mathrm{p}_{1,4}=0,0001\end{array}$ & $\begin{array}{l}\mathrm{p}_{2,3}=0,001 \\
\mathrm{p}_{2,4}=0,001 \\
\mathrm{p}_{3,4}=0,425\end{array}$ & $\begin{array}{l}\mathrm{p}_{1,2}=0,019 \\
\mathrm{p}_{1,3}=0,0001 \\
\mathrm{p}_{1,4}=0,0001\end{array}$ & $\begin{array}{l}\mathrm{p}_{2,3}=0,012 \\
\mathrm{p}_{2,4}=0,001 \\
\mathrm{p}_{3,4}=0,321\end{array}$ & $\begin{array}{l}\mathrm{p}_{1,2}=0,008 \\
\mathrm{p}_{1,3}=0,0001 \\
\mathrm{p}_{1,4}=0,0001\end{array}$ & $\begin{array}{l}\mathrm{p}_{2,3}=0,020 \\
\mathrm{p}_{2,4}=0,399 \\
\mathrm{p}_{3,4}=0,499\end{array}$ & \\
\hline
\end{tabular}

Уровень осведомленности о наличии АГ среди обследованных врачей в нашем исследовании был несколько ниже $(67,15 \%)$ чем в среднем по России $(73,1 \%)$ [2], среди обследованных лиц с АГ в Красноярском крае $(77,9 \%)$ [3, с. 551-559] и исследовании врачей в рамках проекта «Здоровье и образование врача» (84\%) [12, с. 137-144].

Таблица 4.

Осведомленность о наличии АГ среди мужчин и женщин

в зависимости от возраста

\begin{tabular}{|c|c|c|c|c|c|c|c|}
\hline \multirow{2}{*}{\begin{tabular}{|c} 
Воз- \\
растные \\
группы \\
(лет)
\end{tabular}} & \multicolumn{2}{|c|}{ Всего } & \multicolumn{2}{|c|}{ Мужчины } & \multicolumn{2}{|c|}{ Женщины } & \multirow[b]{2}{*}{$p$} \\
\hline & Абс. & $\%$ & Абс. & $\%$ & Абс. & $\%$ & \\
\hline 1. $20-29$ & 4 & 40,0 & 3 & 33,3 & 1 & 100,0 & $\begin{array}{l}p_{\mathrm{M}, \mathrm{K}}= \\
0,400\end{array}$ \\
\hline 2. 30-39 & 10 & 41,7 & 9 & 42,9 & 1 & 33,3 & $\begin{array}{l}p_{\text {M,K }}= \\
0,044\end{array}$ \\
\hline 3. $40-49$ & 25 & 62,5 & 16 & 57,1 & 9 & 75,0 & $\begin{array}{l}p_{\mathrm{M}, \mathrm{K}}= \\
0,090\end{array}$ \\
\hline 4. $50-59$ & 28 & 80,0 & 11 & 73,3 & 17 & 85,0 & $\begin{array}{l}p_{\mathrm{M}, \mathrm{k}}= \\
0,006\end{array}$ \\
\hline
\end{tabular}


Окончание табл. 4.

\begin{tabular}{|c|c|c|c|c|c|c|c|}
\hline $\begin{array}{l}5.60 \text { и } \\
\text { старше }\end{array}$ & 25 & 89,3 & 9 & 75,0 & 16 & 100,0 & $\begin{array}{l}p_{\mathrm{M}, \mathrm{\kappa}}= \\
0,044\end{array}$ \\
\hline 6. Всего & 92 & 67,1 & 47 & 55,3 & 45 & 86,5 & $\begin{array}{l}p_{\mathrm{M}, \mathrm{K}}= \\
0,001\end{array}$ \\
\hline$p$ & $\begin{array}{l}\mathrm{p}_{1,2}=0,617 \\
\mathrm{p}_{1,3}=0,176 \\
\mathrm{p}_{1,4}=0,019 \\
\mathrm{p}_{1,5}=0,004 \\
\mathrm{p}_{2,3}=0,087\end{array}$ & $\begin{array}{l}\mathrm{p}_{2,4}=0,003 \\
\mathrm{p}_{2,5}=0,001 \\
\mathrm{p}_{3,4}=0,079 \\
\mathrm{p}_{3,5}=0,012 \\
\mathrm{p}_{4,5}=0,259\end{array}$ & $\begin{array}{l}\mathrm{p}_{1,2}=0,165 \\
\mathrm{p}_{1,3}=0,483 \\
\mathrm{p}_{1,4}=0,542 \\
\mathrm{p}_{1,5}=0,493 \\
\mathrm{p}_{2,3}=0,129\end{array}$ & $\begin{array}{l}\mathrm{p}_{2,4}=0,007 \\
\mathrm{p}_{2,5}=0,005 \\
\mathrm{p}_{3,4}=0,064 \\
\mathrm{p}_{3,5}=0,044 \\
\mathrm{p}_{4,5}=0,515\end{array}$ & $\begin{array}{l}\mathrm{p}_{1,2}=0,505 \\
\mathrm{p}_{1,3}=0,571 \\
\mathrm{p}_{1,4}=0,210 \\
\mathrm{p}_{1,5}=0,178 \\
\mathrm{p}_{2,3}=0,129\end{array}$ & $\begin{array}{l}\mathrm{p}_{2,4}=0,007 \\
\mathrm{p}_{2,5}=0,005 \\
\mathrm{p}_{3,4}=0,064 \\
\mathrm{p}_{3,5}=0,044 \\
\mathrm{p}_{4,5}=0,515\end{array}$ & \\
\hline
\end{tabular}

Анализ осведомленности о АГ среди врачей различных специальностей показал, что осведомлены о наличии АГ 76,7\% [95\%ДИ: 64,1-76,7] врачей терапевтических отделений, 58,4\% [95\%ДИ: 46,5-58,5] врачей хирургических отделений, 70,0\% [95\%ДИ: 49,9-94,0] анестезиологов-реаниматологов, 77,0\% [95\%ДИ: 50,6-77,8] врачей лучевой диагностики, при этом во всех отделениях отмечалась большая осведомленность у женщин в сравнении с мужчинами (табл. 5).

При анализе приверженности 137 врачей приему антигипертензивных препаратов (АГП) с использованием опросника Мориски- Грина оказалось, что комплаентны лечению 46,7\% [95\%ДИ: 38,56-55,05] (табл. 6). При этом количество женщин врачей с максимальным баллом комплаентности (4) составило 63,5\% [95\%ДИ: 50,4-63,5], мужчин 36,5\% [95\%ДИ: 26,2-36,5] соответственно.

Таблицуа 5.

Осведомленность о наличии АГ у врачей различных специальностей

\begin{tabular}{|c|c|c|c|c|c|c|c|}
\hline \multirow{2}{*}{ Врачи } & \multicolumn{2}{|c|}{ Всего } & \multicolumn{2}{|c|}{ Мужчины } & \multicolumn{2}{|c|}{ Женщины } & \multirow[b]{2}{*}{$p$} \\
\hline & Абс. & $\%$ & Абс. & $\%$ & Абс. & $\%$ & \\
\hline 1. Терапевты & 33 & 76,74 & 5 & 50,00 & 28 & 84,85 & $\begin{array}{c}p_{\mathrm{M}, \mathrm{K}}= \\
0,0001\end{array}$ \\
\hline 2. Хирурги & 38 & 58,46 & 29 & 53,70 & 9 & 81,82 & $\begin{array}{l}p_{\mathrm{M}, \mathrm{K}}= \\
0,0001\end{array}$ \\
\hline $\begin{array}{l}\text { 3. Анестези- } \\
\text { олог. и реа- } \\
\text { ниматолог. }\end{array}$ & 14 & 70,00 & 8 & 61,54 & 6 & 85,71 & $\begin{array}{c}p_{\mathrm{M}, \mathrm{K}}= \\
0,370\end{array}$ \\
\hline $\begin{array}{l}\text { 4. Лучевой } \\
\text { диагностики }\end{array}$ & 7 & 77,78 & 6 & 75,00 & 1 & 100,00 & $\begin{array}{l}p_{\mathrm{M}, \mathrm{K}}= \\
0,025\end{array}$ \\
\hline$p$ & $\begin{array}{l}\mathrm{p}_{1,2}=0,038 \\
\mathrm{p}_{1,3}=0,396 \\
\mathrm{p}_{1,4}=0,559\end{array}$ & $\begin{array}{l}\mathrm{p}_{2,3}=0,255 \\
\mathrm{p}_{2,4}=0,231 \\
\mathrm{p}_{3,4}=0,517\end{array}$ & $\begin{array}{l}\mathrm{p}_{1,2}=0,0002 \\
\mathrm{p}_{1,3}=0,014 \\
\mathrm{p}_{1,4}=0,001\end{array}$ & $\begin{array}{l}\mathrm{p}_{2,3}=0,460 \\
\mathrm{p}_{2,4}=0,188 \\
\mathrm{p}_{3,4}=0,177\end{array}$ & $\begin{array}{l}\mathrm{p}_{1,2}=0,0001 \\
\mathrm{p}_{1,3}=0,009 \\
\mathrm{p}_{1,4}=0,004\end{array}$ & $\begin{array}{l}\mathrm{p}_{2,3}=0,096 \\
\mathrm{p}_{2,4}=0,649 \\
\mathrm{p}_{3,4}=0,273\end{array}$ & \\
\hline
\end{tabular}


Регулярно лечатся 21,2\% [95\%ДИ: 14,3-21,2], нерегулярно 18,2\% [95\%ДИ: 11,8-18,2], а адекватно лечатся всего 28,5\% [95\%ДИ: 20,9-28,5]. Отказ от лечения составил $21,9 \%$ [95\%ДИ: 14,9-21,9]. Только около трети обследованных мужчин с АГ принимали АГП регулярно 14,1\% [95\%ДИ: 6,72-14,12], адекватно 20,0\% [95\%ДИ: 11,0\%-27,1\%]. Женщины врачи в два раза чаще лечатся регулярно 32,7\% [95\%ДИ: 10,4\%-32,7\%] (p=0,009) и адекватно 42,3\% [95\%ДИ: $21,6 \%-42,3 \%](\mathrm{p}=0,005)$ в сравнении с мужчинами (табл. 6).

Таблицуа 6.

Показатели приверженности

к лечению артериальной гипертонии среди врачей

\begin{tabular}{|c|c|c|c|c|c|c|c|c|c|c|}
\hline \multirow{2}{*}{ Группы } & \multicolumn{2}{|c|}{ Лечатся } & \multicolumn{2}{|c|}{ Регулярно } & \multicolumn{2}{|c|}{ Нерегулярно } & \multicolumn{2}{|c|}{ Адекватно } & \multicolumn{2}{|c|}{ Отказ } \\
\hline & Абс. & $\%$ & Абс. & $\%$ & Абс. & $\%$ & Абс. & $\%$ & Абс. & $\%$ \\
\hline 1. Мужчины & 31 & 36,5 & 12 & 14,1 & 14 & 16,5 & 17 & 20,0 & 19 & 22,4 \\
\hline 2. Женщины & 33 & 63,5 & 17 & 32,7 & 11 & 21,2 & 22 & 42,3 & 11 & 21,2 \\
\hline 3. Всего & 64 & 46,7 & 29 & 21,2 & 25 & 18,2 & 39 & 28,5 & 30 & 21,9 \\
\hline$p$ & \multicolumn{2}{|c|}{$\mathrm{p}_{1-2}=0,002$} & \multicolumn{2}{|c|}{$\mathrm{p}_{1-2}=0,009$} & \multicolumn{2}{|c|}{$\mathrm{p}_{1-2}=0,319$} & \multicolumn{2}{|c|}{$\mathrm{p}_{1-2}=0,005$} & \multicolumn{2}{|c|}{$\mathrm{p}_{1-2}=0,523$} \\
\hline
\end{tabular}

В настоящем исследовании эффективность контроля АГ среди врачей оказалась несколько ниже в сравнение с данными, полученными Ротарь О.П. с соавт. (2017) при проведении скрининга по выявлению сердечно-сосудистых факторов риска среди участников конгресса кардиологов 2016 г в Екатеринбурге, где прием АГП составил 63\% [21, с. 126-131], а также в сравнении с результатами исследований Шальновой С.А. с соавт. (2008) согласно которым 61,7\% врачей-мужчин и 79,6\% врачей-женщин с АГ принимают антигипертензивные препараты (АГП), при этом контролируют АГ $20 \%$ среди мужчин и $39,4 \%$ среди женщин [25, с. 28-32].

При изучении отношения к лечению в зависимости от возраста отмечено, что в возрастной группе 20-29 лет получают лечение всего 10\% (табл. 7). В дальнейшем этот показатель увеличивается и достигает максимума в возрастной группе 60 лет и старше (67,9\% 95\%ДИ: 50,56-67,86).

При изучении приверженности к лечению среди врачей различных специальностей установлено, что врачи хирургических отделений имели самые низкие показатели комплаентности, в сравнении с врачами других специальностей (табл. 8). Принимали АГП 36,9\% [95\%ДИ: 26,23-49,08] обследованных врачей хирургов, регулярно 13,8\% [95\%ДИ: 5,45-13,85], адекватно 15,4\% [95\%ДИ: 6,61-15,38], нерегулярно 21,5\% [95\%ДИ: 11,54$21,54]$, отказавшихся от лечения 21,5\% [95\%ДИ: 11,54-21,54]. Среди вра- 
чей различных специальностей чаще лечатся терапевты 60,5\% [95\%ДИ: 45,85-60,47] в сравнении с хирургами - 36,9\% [95\%ДИ: 25,19-36,92], анестезиологами-реаниматологами - 45,0\% [95\%ДИ: 11,98-44,44] и врачами лучевой диагностики - 55,6 [95\%ДИ: 11,76-76,93] соответственно. Наши данные о приверженности к лечению среди врачей различных специальностей были выше, в сравнении с данными полученными Кобалавой Ж.Д. и соавт. (2010), согласно которых пропорция врачей с максимальным баллом комплаентности (4) колебалась от $22,2 \%$ среди хирургов до $38,3 \%$ среди терапевтов [14, с. 12-24].

Таблицуа 7.

Показатели приверженности к лечению артериальной гипертонии среди врачей различных возрастных групп

\begin{tabular}{|l|c|c|c|c|c|c|c|c|c|c|}
\hline \multirow{2}{*}{$\begin{array}{c}\text { Возрастные } \\
\text { группы }\end{array}$} & \multicolumn{2}{|c|}{ Лечатся } & \multicolumn{2}{c|}{ Регулярно } & \multicolumn{2}{c|}{ Нерегулярно } & \multicolumn{2}{|c|}{ Адекватно } & \multicolumn{2}{c|}{ Отказ } \\
\cline { 2 - 13 } & Абс. & $\%$ & Абс. & $\%$ & Абс. & $\%$ & Абс. & $\%$ & Абс. & $\%$ \\
\hline $1.20-29$ & 1 & 10,0 & 1 & 10,0 & 0 & 0,0 & 1 & 10,0 & 3 & 30,0 \\
\hline $2.30-39$ & 7 & 29,2 & 2 & 8,3 & 5 & 20,8 & 2 & 8,3 & 3 & 12,5 \\
\hline $3.40-49$ & 18 & 45,0 & 9 & 22,5 & 6 & 15,0 & 12 & 30,0 & 9 & 22,5 \\
\hline $4.50-59$ & 19 & 54,3 & 11 & 31,4 & 6 & 17,1 & 13 & 37,1 & 9 & 25,7 \\
\hline 5.60 и старше & 19 & 67,9 & 6 & 21,4 & 8 & 28,6 & 11 & 39,3 & 6 & 21,4 \\
\hline & $\mathrm{p}_{1-2}=0,231$ & $\mathrm{p}_{1-2}=0,662$ & $\mathrm{p}_{1-2}=0,153$ & $\mathrm{p}_{1-2}=0,662$ & $\mathrm{p}_{1-2}=0.228$ \\
& $\mathrm{p}_{1-3}=0,042$ & $\mathrm{p}_{1-3}=0,349$ & $\mathrm{p}_{1-3}=0,241$ & $\mathrm{p}_{1-3}=0,191$ & $\mathrm{p}_{1-3}=0,449$ \\
& $\mathrm{p}_{1-4}=0,014$ & $\mathrm{p}_{1-4}=0,174$ & $\mathrm{p}_{1-4}=0,199$ & $\mathrm{p}_{1-4}=0,102$ & $\mathrm{p}_{1-4}=0,539$ \\
& $\mathrm{p}_{1-5}=0,002$ & $\mathrm{p}_{1-5}=0,392$ & $\mathrm{p}_{1-5}=0,063$ & $\mathrm{p}_{1-5}=0,090$ & $\mathrm{p}_{1-5}=0,440$ \\
& $\mathrm{p}_{2-3}=0,161$ & $\mathrm{p}_{2-3}=0,132$ & $\mathrm{p}_{2-3}=0,392$ & $\mathrm{p}_{2-3}=0,039$ & $\mathrm{p}_{2-3}=0,258$ \\
& $\mathrm{p}_{2-4}=0,049$ & $\mathrm{p}_{2-4}=0,034$ & $\mathrm{p}_{2-4}=0,488$ & $\mathrm{p}_{2-4}=0,011$ & $\mathrm{p}_{2-4}=0,183$ \\
& $\mathrm{p}_{2-5}=0,006$ & $\mathrm{p}_{2-5}=0,180$ & $\mathrm{p}_{2-5}=0,375$ & $\mathrm{p}_{2-5}=0,010$ & $\mathrm{p}_{2-5}=0,318$ \\
& $\mathrm{p}_{3-4}=0,284$ & $\mathrm{p}_{3-4}=0,270$ & $\mathrm{p}_{3-4}=0,523$ & $\mathrm{p}_{3-4}=0,341$ & $\mathrm{p}_{3-4}=0,477$ \\
& $\mathrm{p}_{3-5}=0,052$ & $\mathrm{p}_{3-5}=0,579$ & $\mathrm{p}_{3-5}=0,145$ & $\mathrm{p}_{3-5}=0,295$ & $\mathrm{p}_{3-5}=0,361$ \\
& $\mathrm{p}_{4-5}=0,202$ & $\mathrm{p}_{4-5}=0,275$ & $\mathrm{p}_{4-5}=0,218$ & $\mathrm{p}_{4-5}=0,533$ & $\mathrm{p}_{4-5}=0,463$ \\
\hline
\end{tabular}

Низкие уровни комплаентности среди врачей хирургического профиля в нашем исследовании можно объяснить преобладанием лиц мужского пола, среди которых исходно показатели приверженности были ниже в сравнении с женщинами, а также спецификой работы. Хирургам в большей степени по сравнению с терапевтами свойственны высокая производственная нагрузка, круглосуточный режим работы с обязательными дежурствами, контакт с умирающими больными, психоэмоциональное напряжение $[1$, с. 52-5]. По данным исследования Кобалавы Ж.Д. и соавт. (2010) среди хирургов максимальна частота АГ и курения, более трети $36,2 \%$, характеризуются высоким риском сердечно-сосудистой смерти по 
SCORE [14, с. 12-24]. Хирурги наименее информированы о состоянии своих ФР, значительно реже принимают АГП, меньше контролируют АД и существенно реже, чем врачи других специальностей, склонны продолжать рекомендованную медикаментозную терапию [14, с. 12-24]. Таким образом, профессиональную деятельность хирургов можно отнести к факторам риска.

Таблийа 8.

Показатели приверженности к лечению артериальной гипертонии среди врачей различных специальностей

\begin{tabular}{|c|c|c|c|c|c|c|c|c|c|c|}
\hline \multirow{2}{*}{$\begin{array}{c}\text { Возрастные } \\
\text { группы }\end{array}$} & \multicolumn{2}{|c|}{ Лечатся } & \multicolumn{2}{|c|}{ Регулярно } & \multicolumn{2}{|c|}{ Нерегулярно } & \multicolumn{2}{|c|}{ Адекватно } & \multicolumn{2}{|c|}{ Отказ } \\
\hline & Абс. & $\%$ & Абс. & $\%$ & Абс. & $\%$ & Абс. & $\%$ & Абс. & $\%$ \\
\hline 1. Терапевты & 26 & 60,5 & 14 & 32,6 & 8 & 18,6 & 18 & 41,9 & 9 & 20,9 \\
\hline 2. Хирурги & 24 & 36,9 & 9 & 13,8 & 10 & 15,4 & 14 & 21,5 & 14 & 21,5 \\
\hline $\begin{array}{l}\text { 3. Анестезио- } \\
\text { лог. и реанима- } \\
\text { толог. }\end{array}$ & 9 & 45,0 & 4 & 20,0 & 4 & 20,0 & 5 & 25,0 & 5 & 25,0 \\
\hline $\begin{array}{l}\text { 4. Лучевой диа- } \\
\text { гностики }\end{array}$ & 5 & 55,6 & 2 & 22,2 & 3 & 33,3 & 2 & 22,2 & 2 & 22,2 \\
\hline 5. Всего & 64 & 46,7 & 29 & 21,2 & 25 & 18,2 & 39 & 28,5 & 29 & 21,2 \\
\hline$p$ & $\begin{array}{l}\mathrm{p}_{1-2}=0 \\
\mathrm{p}_{1-3}=0 \\
\mathrm{p}_{1-4}=0 \\
\mathrm{p}_{2-3}=0 \\
\mathrm{p}_{2-4}=0 \\
\mathrm{p}_{3-4}=0\end{array}$ & $\begin{array}{l}, 014 \\
, 190 \\
533 \\
347 \\
, 225 \\
499 \\
\end{array}$ & $\begin{array}{l}\mathrm{p}_{1-2}= \\
\mathrm{p}_{1-3}= \\
\mathrm{p}_{1-4}= \\
\mathrm{p}_{2-3}= \\
\mathrm{p}_{2-4}= \\
\mathrm{p}_{3-4}=\end{array}$ & $\begin{array}{l}, 019 \\
, 236 \\
429 \\
362 \\
400 \\
, 624\end{array}$ & $\begin{array}{l}\mathrm{p}_{1-2}= \\
\mathrm{p}_{1-3}= \\
\mathrm{p}_{1-4}= \\
\mathrm{p}_{2-3}= \\
\mathrm{p}_{2-4}= \\
\mathrm{p}_{3-4}=\end{array}$ & $\begin{array}{l}426 \\
574 \\
283 \\
427 \\
189 \\
369 \\
\end{array}$ & $\begin{array}{l}\mathrm{p}_{1-2}= \\
\mathrm{p}_{1-3}= \\
\mathrm{p}_{1-4}= \\
\mathrm{p}_{2-3}= \\
\mathrm{p}_{2-4}== \\
\mathrm{p}_{3-4}=\end{array}$ & $\begin{array}{l}021 \\
156 \\
239 \\
480 \\
626 \\
631 \\
\end{array}$ & $\begin{array}{l}\mathrm{p}_{1-2}=0 \\
\mathrm{p}_{1-3}=0 \\
\mathrm{p}_{1-4}=0 \\
\mathrm{p}_{2-3}=0 \\
\mathrm{p}_{2-4}=0 \\
\mathrm{p}_{3-4}=0\end{array}$ & $\begin{array}{l}, 569 \\
, 476 \\
, 619 \\
, 480 \\
, 626 \\
, 631 \\
\end{array}$ \\
\hline
\end{tabular}

\section{Заключение}

Резюмируя вышеизложенное, следует отметить, что среди обследованных врачей частота встречаемости АГ составила 43,22\% и не превышала среднероссийский уровень. Данный показатель был существенно выше среди мужчин врачей, в сравнении с женщинами. Указанное гендерное отличие особенно выражено в возрастной группе 20-29 лет и 30-39 лет. Среди анестезиологов, реаниматологов и врачей лучевой диагностики АГ встречалась чаще по сравнению с врачами других специальностей. У врачей не зависимо от пола чаще отмечалась АГ 1 степени. Несмотря на достаточно высокую информированность о методах профилактики и лечения сердечно-сосудистых заболеваний, полученных в рамках профессионального образования, показатели приверженности к лечению АГ были ниже 
среднероссийского уровня. Врачи хирургических отделений имели самые низкие показатели осведомленности и комплаентности, в сравнении с врачами других специальностей.

\section{Список литературы}

1. Барбараш О.Л., Башева Л.И., Смакотина С.А. и др. Факторы сердечно-сосудистого риска у врачей различных специальностей // Кардиология. 2008. №7. C. 52-5.

2. Бойцов С.А., Баланова Ю.А., Шальнова С.А. и др. Артериальная гипертония среди лиц 25-64 лет: распространенность, осведомленность, лечение и контроль. По материалам исследования ЭССЕ. Кардиоваскулярная терапия и профилактика. 2014. Т.14, №4. С. 4-14.

3. Гринштейн Ю.И., Петрова М.М., Шабалин В.В. и др. Распространенность артериальной гипертензии в Красноярском крае по данным эпидемиологического исследования ЭССЕ-РФ // Артериальная гипертензия. 2016. №6. С. 551-559.

4. Давыдов Е.Л. Яскевич Р.А., Кусаев В.В. и др. Проблемы приверженности к лечению пациентов старших возрастных групп с артериальной гипертонией в г. Красноярске в рамках организационно-функциональной модели медико-социальной помощи // Международный журнал экспериментального образования. 2016. № 5-3. С. 285-289.

5. Давыдов Е.Л., Яскевич Р.А., Мажаров В.Ф. Факторы, препятствующие приобретению пациентами старших возрастных групп лекарственных средств для лечения артериальной гипертонии // Международный журнал экспериментального образования. 2016. № 5-3. С. 290-295.

6. Деревянных Е.В., Балашова Н.А., Яскевич Р.А., Москаленко О.Л. Анализ распространенности факторов риска сердечно-сосудистых заболеваний среди мужчин медицинских работников крупной клинической больницы города Красноярска // В мире научных открытий. 2017. Т. 9. № 4. С. 10-33.

7. Деревянных Е.В., Поликарпов Л.С., Петрова М.М. Частота артериальной гипертонии среди женщин - медицинских работников крупного промышленного города // Сибирское медицинское обозрение. 2008. № 4 (52). C. $89-92$.

8. Деревянных Е.В., Поликарпов Л.С., Петрова М.М. Частота артериальной гипертонии среди женщин медицинских работников клинической больницы скорой медицинской помощи города Красноярска // Вестник РУДН. Серия: Медицина. 2008. №7. С. 130-139. 
9. Деревянных Е.В., Яскевич Р.А., Балашова Н.А., Москаленко О.Л. Распространенность избыточной массы тела среди женщин медицинских работников крупной клинической больницы города Красноярска // В мире научных открытий. 2016. № 7 (79). С. 26-46.

10. Деревянных Е.В., Яскевич Р.А., Балашова Н.А. Возрастные особенности приверженности к лечению артериальной гипертонии у мужчин г. Красноярска // Международный журнал прикладных и фундаментальных исследований. 2016. № 6-1. С. 48-52.

11. Деревянных Е.В., Яскевич Р.А. Возрастные особенности приверженности к лечению артериальной гипертонии среди медицинских работников крупной клинической больницы города Красноярска // Клиническая геронтология. 2017. Т. 23. № 9-10. С. 21-23.

12. Дроздова Л.Ю., Марцевич С.Ю., Воронина В.П. Одновременная оценка распространенности и эффективности коррекции факторов риска сердечно-сосудистых заболеваний среди врачей и их знания современных клинических рекомендаций. Результаты проекта «Здоровье и образование врача» // Рациональная фармакотерапия в кардиологии. 2011. №7(2). С. 137-144.

13. Карамнова Н.С., Калинина А.М., Григорян Ц.А. и др. Распространенность факторов, формирующих суммарный кардиоваскулярный риск среди медицинских работников первичного звена здравоохранения // Кардиоваскулярная терапия и профилактика. 2009. Т. 8, № 6. С. 54-58.

14. Кобалава Ж.Д., Котовская Ю.В., Шальнова С.А. Сердечно-сосудистый риск у врачей разных специальностей. Результаты Российской многоцентровой научно-образовательной программы «Здоровье врачей России» // Кардиоваскулярная терапия и профилактика. 2010. № 4. С. 12-24.

15. Найденова Н.Е., Лобыкина Е.Н. Распространенность факторов риска неинфекционных заболеваний у медицинских работников первичного звена здравоохранения Томской области // Профилактическая медицина. 2015. T.18. № 2. С. 38-42.

16. Неврычева Е.В., Жмеренецкий К.В., Ноздрина Н.С. Здоровье медицинских работников // Здравоохранение Дальнего Востока. 2016. № 1 (67). C. $72-82$.

17. Поликарпов Л.С., Хамнагадаев И.И., Иванова Е.Б. и др. Частота сердечно-сосудистой патологии, содержание микроэлементов в различных средах в условиях Севера // Сибирский медицинский журнал (г. Томск). 2005. T.20, № 2. C. 55-57.

18. Поликарпов Л.С., Хамнагадаев И.И. Манчук В.Т. и др. Социально-эпидемиологическая характеристика артериальной гипертонии в условиях 
Севера и Сибири // Сибирское медицинское обозрение. 2008. № 4(52). C. $92-95$.

19. Поликарпов Л.С., Хамнагадаев И.И., Яскевич Р.А., Деревянных Е.В. Артериальная гипертония (распространенность, профилактика, адаптация и реадаптация к различным экологическим условиям. Красноярск: Издательство КрасГМУ, 2010. 289 с.

20. Поликарпов Л.С., Деревянных Е.В., Яскевич Р.А., Балашова Н.А. Особенности приверженности к лечению артериальной гипертонии среди женщин пожилого возраста // Современные проблемы науки и образования. 2014. № 5. URL: http://www.science-education.ru/ru/article/view?id=14899

21. Ротарь О.П., Орлов А.В., Бояринова М.А. и др. Здоровье кардиологов: кто, если не они? // РКЖ. 2017. №5 (145). С. 126-131.

22. Терентьев В.П., Чесникова А.И., Левицкая Е.С., Хлиян К.Г. Гендерные особенности состояния здоровья врачей: когортное исследование (на примере г. Ростова-на-дону и области) // Проблемы женского здоровья. 2011. T. 6. № 2. С. 5-9.

23. Филимонова Л.А., Яскевич Р.А., Давыдов Е.Л. Вопросы формирования и течения артериальной гипертонии в пожилом и старческом возрасте // Современные проблемы науки и образования. 2016. № 6. http://scienceeducation.ru/ru/article/view?id=25458

24. Хамнагадаев И.И., Яскевич Р.А., Поликарпов Л.С., Новгородцева Н.Я. Распространенность артериальной гипертонии и избыточной массы тела среди сельского населения северных регионов // Сибирский медицинский журнал (г. Томск). 2004. Т.19. №4. С. 94-96.

25. Шальнова С.А., Оганов Р.Г., Деев А.Д., Кукушкин С.К. Здоровье российских врачей. Клинико-эпидемиологический анализ // Кардиоваскулярная терапия и профилактика. 2008. Т. 7. № 6. С. 28-32.

26. Яскевич Р.А., Москаленко О.Л. Анализ частоты и структуры заболеваний сердечно-сосудистой системы у мигрантов Крайнего Севера в период реадаптации к новым климатическим условиям // В мире научных открытий. 2017. T. 9. № 4-2. С. 41-58.

27. Яскевич Р.А. Особенности клинического течения артериальной гипертонии у мигрантов Крайнего Севера в период реадаптации к новым климатическим условиям // Кардиология на перекрестке наук Тезисы докладов. 2017. C. $346-348$.

28. Artyukhov I.P., Grinshtein Y.I., Petrova M.M. et al. Prevalence of arterial hypertension in the Krasnoyarsk Krai (Siberia, Russia) // BMC Cardiovascular Disorders. 2017. 17:138. 
29. Bolbrinker J, Zaidi Touis L, Gohlke H, Weisser B, Kreutz R. European guidelines on lifestyle changes for management of hypertension: Awareness and implementation of recommendations among German and European physicians. Herz. 2017 May 22. doi: 10.1007/s00059-017-4575-0.

30. Dayoub E, Jena AB. Chronic Disease Prevalence and Healthy Lifestyle Behaviors Among U.S. Health Care Professionals. Mayo Clinic proceedings. 2015. 90(12), pp. 1659-1662.

31. Lerssrimongkol C., Wisetborisut A., Angkurawaranon C. et al. Active commuting and cardiovascular risk among health care workers // Occup Med (Lond). 2016 Aug. 66(6):483-7.

32. World Health Organization. Cardiovascular diseases (CVDs) - Fact sheet. 2017. [Cited 4 Sep 2017.] URL: http://www.who.int/mediacentre/factsheets/ fs $317 / \mathrm{en} /$

33. Yaskevich R.A., Moskalenko O.L. Analysis of the incidence and structure of the cardiovascular system diseases in the far north migrants over the period of readaptation to the new climatic conditions // В мире научных открытий. 2017. T. 9. № 4-2, pp. 59-73.

34. Москаленко О.Л. Влияние городского техногенного загрязнения на морфофункциональное состояние юношей: автореф. дисс. канд. биол. наук. Красноярск, 2014. 18 с.

\section{References}

1. Barbarash O.L., Basheva L.I., Smakotina S.A. i dr. Faktory serdechno-sosudistogo riska u vrachey razlichnykh spetsial'nostey [Factors of cardiovascular risk in physicians of various specialties]. Kardiologiya [Cardiology]. 2008. №7, pp. 52-5.

2. Boytsov S.A., Balanova Yu.A., Shal'nova S.A. i dr. Arterial'naya gipertoniya sredi lits 25-64 let: rasprostranennost', osvedomlennost', lechenie i kontrol'. Po materialam issledovaniya ESSE [Arterial hypertension among persons aged 25-64 years: prevalence, awareness, treatment and control. Based on the materials of the ESSE study]. Kardiovaskulyarnaya terapiya i profilakti$k a$ [Cardiovascular therapy and prevention]. 2014. V.14. №4, pp. 4-14.

3. Grinshteyn Yu.I., Petrova M.M., Shabalin V.V. i dr. Rasprostranennost' arterial'noy gipertenzii v Krasnoyarskom krae po dannym epidemiologicheskogo issledovaniya ESSE-RF [The prevalence of arterial hypertension in the Krasnoyarsk Territory according to the epidemiological study of the ESSE-RF]. Arterial'naya gipertenziya [Arterial hypertension]. 2016. №, pp. 551-559. 
4. Davydov E.L. Yaskevich R.A., Kusaev V.V. et al. Problemy priverzhennosti $\mathrm{k}$ lecheniyu patsientov starshikh vozrastnykh grupp s arterial'noy gipertoniey v g. Krasnoyarske v ramkakh organizatsionno-funktsional'noy modeli mediko-sotsial'noy pomoshchi [Problems of adherence to the treatment of patients of older age groups with arterial hypertension in Krasnoyarsk within the framework of the organizational and functional model of medical and social care]. Mezhdunarodnyy zhurnal eksperimental'nogo obrazovaniya [International Journal of Experimental Education]. 2016. № 5-3, pp. 285-289.

5. Davydov E.L., Yaskevich R.A., Mazharov V.F. Faktory, prepyatstvuyushchie priobreteniyu patsientami starshikh vozrastnykh grupp lekarstvennykh sredstv dlya lecheniya arterial'noy gipertonii [Factors hindering the acquisition by patients of older age groups of medicines for the treatment of arterial hypertension]. Mezhdunarodnyy zhurnal eksperimental'nogo obrazovaniya [International Journal of Experimental Education]. 2016. № 5-3, pp. 290-295.

6. Derevyannykh E.V., Balashova N.A., Yaskevich R.A., Moskalenko O.L. Analiz rasprostranennosti faktorov riska serdechno-sosudistykh zabolevaniy sredi muzhchin meditsinskikh rabotnikov krupnoy klinicheskoy bol'nitsy goroda Krasnoyarska [An analysis of the prevalence of cardiovascular risk factors among men in the medical clinic of a major clinical hospital in the city of Krasnoyarsk]. V mire nauchnykh otkrytiy [Siberian Journal of Life Sciences and Agriculture]. 2017. V. 9. № 4, pp. 10-33.

7. Derevyannykh E.V., Polikarpov L.S., Petrova M.M. Chastota arterial'noy gipertonii sredi zhenshchin - meditsinskikh rabotnikov krupnogo promyshlennogo goroda [The frequency of arterial hypertension among women - medical workers of a large industrial city]. Sibirskoe meditsinskoe obozrenie [Siberian Medical Review]. 2008. № 4 (52), pp. 89-92.

8. Derevyannykh E.V., Polikarpov L.S., Petrova M.M. Chastota arterial'noy gipertonii sredi zhenshchin meditsinskikh rabotnikov klinicheskoy bol'nitsy skoroy meditsinskoy pomoshchi goroda Krasnoyarska [The frequency of arterial hypertension among women of medical workers in the clinical hospital of the ambulance service of the city of Krasnoyarsk]. Vestnik RUDN. Seriya: Meditsina [Bulletin of the Peoples' Friendship University of Russia. Series: Medicine]. 2008. №7, pp. 130-139.

9. Derevyannykh E.V., Yaskevich R.A., Balashova N.A., Moskalenko O.L. Rasprostranennost' izbytochnoy massy tela sredi zhenshchin meditsinskikh rabotnikov krupnoy klinicheskoy bol'nitsy goroda Krasnoyarska [The prevalence of overweight among women medical workers of a major clinical hospital in the city of Krasnoyarsk]. V mire nauchnykh otkrytiy [Siberian Journal of Life Sciences and Agriculture]. 2016. № 7 (79), pp. 26-46. 
10. Derevyannykh E.V., Yaskevich R.A., Balashova N.A. Vozrastnye osobennosti priverzhennosti $\mathrm{k}$ lecheniyu arterial'noy gipertonii u muzhchin g. Krasnoyarska [Age features of adherence to the treatment of arterial hypertension in men in Krasnoyarsk]. Mezhdunarodnyy zhurnal prikladnykh i fundamental'nykh issledovaniy [International Journal of Applied and Fundamental Research]. 2016. № 6-1, pp. 48-52.

11. Derevyannykh E.V., Yaskevich R.A. Vozrastnye osobennosti priverzhennosti k lecheniyu arterial'noy gipertonii sredi meditsinskikh rabotnikov krupnoy klinicheskoy bol'nitsy goroda Krasnoyarska [Age features of adherence to the treatment of arterial hypertension among medical workers of a major clinical hospital in the city of Krasnoyarsk]. Klinicheskaya gerontologiya [Clinical gerontology]. 2017. V. 23. № 9-10, pp. 21-23.

12. Drozdova L.Yu., Martsevich S.Yu., Voronina V.P. Odnovremennaya otsenka rasprostranennosti i effektivnosti korrektsii faktorov riska serdechno-sosudistykh zabolevaniy sredi vrachey i ikh znaniya sovremennykh klinicheskikh rekomendatsiy. Rezul'taty proekta «Zdorov'e i obrazovanie vracha» [Simultaneous assessment of the prevalence and effectiveness of correction of cardiovascular risk factors among physicians and their knowledge of current clinical recommendations. Results of the project "Health and education of a doctor"]. Ratsional'naya farmakoterapiya $v$ kardiologii [Rational pharmacotherapy in cardiology]. 2011. №7(2), pp. 137-144.

13. Karamnova N.S., Kalinina A.M., Grigoryan Ts.A. i dr. Rasprostranennost' faktorov, formiruyushchikh summarnyy kardiovaskulyarnyy risk sredi meditsinskikh rabotnikov pervichnogo zvena zdravookhraneniya [The prevalence of factors that form the total cardiovascular risk among primary health care providers]. Kardiovaskulyarnaya terapiya i profilaktika [Cardiovascular therapy and prevention]. 2009. V. 8. № 6, pp. 54-58.

14. Kobalava Zh.D., Kotovskaya Yu.V., Shal'nova S.A. Serdechno-sosudistyy risk u vrachey raznykh spetsial'nostey. Rezul'taty Rossiyskoy mnogotsentrovoy nauchno-obrazovatel'noy programmy «Zdorov'e vrachey Rossii» [Cardiovascular risk in doctors of different specialties. Results of the Russian multicenter scientific and educational program "Health of Russian doctors"]. Kardiovaskulyarnaya terapiya i profilaktika [Cardiovascular therapy and prevention]. 2010. № 4, pp. 12-24.

15. Naydenova N.E., Lobykina E.N. Rasprostranennost' faktorov riska neinfektsionnykh zabolevaniy u meditsinskikh rabotnikov pervichnogo zvena zdravookhraneniya Tomskoy oblasti [Prevalence of non-infectious disease risk factors among primary health care providers in the Tomsk region]. Profilakticheskaya meditsina [Preventive medicine]. 2015. V.18. № 2, pp. 38-42. 
16. Nevrycheva E.V., Zhmerenetskiy K.V., Nozdrina N.S. Zdorov'e meditsinskikh rabotnikov [Health of medical workers]. Zdravookhranenie Dal'nego Vostoka [Health of the Far East]. 2016. № 1 (67), pp. 72-82.

17. Polikarpov L.S., Khamnagadaev I.I., Ivanova E.B. i dr. Chastota serdechnososudistoy patologii, soderzhanie mikroelementov $\mathrm{v}$ razlichnykh sredakh $\mathrm{v}$ usloviyakh Severa [The frequency of cardiovascular pathology, the content of trace elements in various environments in the North]. Sibirskiy meditsinskiy zhurnal (g. Tomsk) [Siberian Medical Journal (Tomsk)]. 2005. V.20. № 2, pp. 55-57.

18. Polikarpov L.S., Khamnagadaev I.I. Manchuk V.T. et al. Sotsial'no-epidemiologicheskaya kharakteristika arterial'noy gipertonii v usloviyakh Severa i Sibiri [Socio-epidemiological characteristics of arterial hypertension in the conditions of the North and Siberia]. Sibirskoe meditsinskoe obozrenie [Siberian Medical Review]. 2008. № 4(52), pp. 92-95.

19. Polikarpov L.S., Khamnagadaev I.I., Yaskevich R.A., Derevyannykh E.V. Arterial'naya gipertoniya (rasprostranennost', profilaktika, adaptatsiya i readaptatsiya k razlichnym ekologicheskim usloviyam [Arterial hypertension (prevalence, prophylaxis, adaptation and adaptation to various ecological conditions]. Krasnoyarsk: Izdatel'stvo KrasGMU. 2010. 289 p.

20. Polikarpov L.S., Derevyannykh E.V., Yaskevich R.A., Balashova N.A. Osobennosti priverzhennosti $\mathrm{k}$ lecheniyu arterial'noy gipertonii sredi zhenshchin pozhilogo vozrasta [Features of adherence to the treatment of arterial hypertension among women of advanced age]. Sovremennye problemy nauki i obrazovaniya [Modern problems of science and education]. 2014. №5. URL: http:// www.science-education.ru/ru/article/view?id=14899

21. Rotar' O.P., Orlov A.V., Boyarinova M.A. et al. Zdorov'e kardiologov: kto, esli ne oni? [Health of cardiologists: who, if not they?]. RKZh [RQF]. 2017. №5 (145), pp. 126-131.

22. Terent'ev V.P., Chesnikova A.I., Levitskaya E.S., Khliyan K.G. Gendernye osobennosti sostoyaniya zdorov'ya vrachey: kogortnoe issledovanie (na primere g. Rostova-na-donu i oblasti) [Gender features of the state of health of doctors: cohort study (on the example of Rostov-on-Don and the region)]. Problemy zhenskogo zdorov'ya [Problems of female health]. 2011. V.6. №2, pp. 5-9.

23. Filimonova L.A., Yaskevich R.A., Davydov E.L. Voprosy formirovaniya i techeniya arterial'noy gipertonii v pozhilom i starcheskom vozraste [Questions of formation and course of arterial hypertension in elderly and senile age]. Sovremennye problemy nauki i obrazovaniya [Modern problems of science and education]. 2016. № 6. http://science-education.ru/ru/article/view?id=25458 
24. Khamnagadaev I.I., Yaskevich R.A., Polikarpov L.S., Novgorodtseva N.Ya. Rasprostranennost' arterial'noy gipertonii i izbytochnoy massy tela sredi sel'skogo naseleniya severnykh regionov [The prevalence of arterial hypertension and overweight among rural population of northern regions]. Sibirskiy meditsinskiy zhurnal (g. Tomsk) [Siberian Medical Journal (Tomsk)]. 2004. V.19. №4, pp. 94-96.

25. Shal'nova S.A., Oganov R.G., Deev A.D., Kukushkin S.K. Zdorov'e rossiyskikh vrachey. Kliniko-epidemiologicheskiy analiz [The health of Russian doctors. Clinical and epidemiological analysis]. Kardiovaskulyarnaya terapiya i profilaktika [Cardiovascular therapy and prevention]. 2008. V. 7. №6, pp. 28-32.

26. Yaskevich R.A., Moskalenko O.L. Analiz chastoty i struktury zabolevaniy serdechno-sosudistoy sistemy u migrantov Kraynego Severa v period readaptatsii $\mathrm{k}$ novym klimaticheskim usloviyam [Analysis of the frequency and structure of diseases of the cardiovascular system in the migrants of the Far North in the period of adaptation to new climatic conditions]. V mire nauchnykh otkrytiy [Siberian Journal of Life Sciences and Agriculture]. 2017. V. 9. № 4-2, pp. 41-58.

27. Yaskevich R.A. Osobennosti klinicheskogo techeniya arterial'noy gipertonii u migrantov Kraynego Severa v period readaptatsii k novym klimaticheskim usloviyam [Peculiarities of the clinical course of arterial hypertension in the migrants of the Far North in the period of readaptation to new climatic conditions]. Kardiologiya na perekrestke nauk Tezisy dokladov [Cardiology at the Crossroads of Sciences. Abstracts]. 2017, pp. 346-348.

28. Artyukhov I.P., Grinshtein Y.I., Petrova M.M. et al. Prevalence of arterial hypertension in the Krasnoyarsk Krai (Siberia, Russia). BMC Cardiovascular Disorders. 2017. 17:138.

29. Bolbrinker J., Zaidi Touis L., Gohlke H., Weisser B., Kreutz R. European guidelines on lifestyle changes for management of hypertension: Awareness and implementation of recommendations among German and European physicians. Herz. 2017 May 22. doi: 10.1007/s00059-017-4575-0.

30. Dayoub E, Jena AB. Chronic Disease Prevalence and Healthy Lifestyle Behaviors Among U.S. Health Care Professionals. Mayo Clinic proceedings. 2015. 90(12), pp. 1659-1662.

31. Lerssrimongkol C., Wisetborisut A., Angkurawaranon C. et al. Active commuting and cardiovascular risk among health care workers. Occup Med (Lond). 2016 Aug. 66(6):483-7.

32. World Health Organization. Cardiovascular diseases (CVDs) - Fact sheet. 2017. [Cited 4 Sep 2017]. http://www.who.int/mediacentre/factsheets/fs317/en/

33. Yaskevich R.A., Moskalenko O.L. Analysis of the incidence and structure of the cardiovascular system diseases in the far north migrants over the period of 
readaptation to the new climatic conditions. V mire nauchnykh otkrytiy [Siberian Journal of Life Sciences and Agriculture]. 2017. V.9. № 4-2, pp. 59-73.

34. Moskalenko O.L. Vliyanie gorodskogo tekhnogennogo zagryazneniya na morfofunktsional'noe sostoyanie yunoshey [Influence of urban anthropogenic pollution on the morphofunctional state of youth]. Krasnoyarsk. 2014. 18 p.

\section{ДАННЫЕ ОБ АВТОРАХ}

Деревянных Евгений Валерьевич, доцент кафедры пропедевтики внутренних болезней и терапии, кандидат медицинских наук ГБОУ ВПО «КрасГМУ им. проф. Ф.В. Войно-Ясенеикого» МЗ РФ ул. Партизана Железняка, 1а, г. Красноярск, 660022, Российская Федераиция rusene@mail.ru

Балашова Наталья Арленовна, доцент кафедры пропедевтики внутренних болезней и терапии, кандидат медицинских наук, доцент ГБОУ ВПО «КрасГМУ им. проф. Ф.В. Войно-Ясенеикого» МЗ РФ ул. Партизана Железняка, 1а, г. Красноярск, 660022, Российская Федерация balashova-61@mail.ru

Яскевич Роман Анатольевич, доцент кафедры пропедевтики внутренних болезней и терапии, ведущий научный сотрудник группы патологии сердечно-сосудистой системы, кандидат медицинских наук, доцент ГБОУ ВПО «КрасГМУ им. проф. Ф.В. Войно-Ясенеикого» МЗ РФ; Федеральное государственное бюджетное научное учреждение «Научно-исследовательский институт медииинских проблем Севера» ул. Партизана Железняка, 1а, г. Красноярск, 660022, Российская Федерация; ул. Партизана Железняка, 32, г. Красноярск, 660022, Российская Федераиия cardio@impn.ru

Москаленко Ольга Леонидовна старший научный сотрудник, кандидат биологических наук Федеральное государственное бюджетное научное учреждение «Научно-исследовательский институт медичинских проблем Севера» ул. Партизана Железняка, 32, г. Красноярск, 660022, Российская Федераиия gre-ll@mail.ru 
DATA ABOUT THE AUTHORS

Derevyannich Evgeny Valeryevich, associate professor at department of propedeutics of internal diseases and therapy, candidate of medical science Krasnoyarsk State Medical University named after Professor V.F. Voino-Yasenetzkiy 1a, P. Zheleznyaka St., Krasnoyarsk, 660022, Russian Federation rusene@mail.ru

Balashova Natalia Arlenovna, associate professor at department of propedeutics of internal diseases and therapy, candidate of medical science, docent

Krasnoyarsk State Medical University named after Professor V.F. Voino-Yasenetzkiy 1a, P. Zheleznyaka St., Krasnoyarsk, 660022, Russian Federation

balashova-61@mail.ru

Yaskevich Roman Anatolyevich, associate professor at department of propedeutics of internal diseases and therapy, leading researcher of the group pathology of the cardiovascular system, candidate of medical science, docent

Krasnoyarsk State Medical University named after Professor V.F. Voino-Yasenetzkiy; Scientific Research Institute of medical problems of the North

1a, P. Zheleznyaka St., Krasnoyarsk, 660022, Russian Federation; 3g, P. Zheleznyaka St., Krasnoyarsk, 660022, Russian Federation cardio@impn.ru

Moskalenko Olga Leonidovna, Senior Researcher, Candidate of Biological Sciences

Scientific Research Institute of medical problems of the North 3g, P. Zheleznyaka St., Krasnoyarsk, 660022, Russian Federation gre-ll@mail.ru 